\title{
Modelo de aprendizaje microcurricular activo: Una guía de planificación áulica para Educación Física
}

\author{
An Active Microcurricular Learning Model: A Guide to Classroom Planning for Physical Education \\ Modelo de aprendizagem micro curricular ativa: um guia de planejamento de classe para \\ educação física
}

Richar Jacobo Posso-Pacheco

Universidad Central del Ecuador

Quito, Ecuador

rjposso@uce.edu.ec

iD https://orcid.org/0000-0003-1279-9852

Laura Cristina Barba-Miranda

Ministerio de Educación del Ecuador

Quito, Ecuador

laura.barba@educacion.gob.ec

https://orcid.org/0000-0002-6902-8066

Ángel Freddy Rodríguez-Torres Universidad Central del Ecuador

Quito, Ecuador afrodriguez@uce.edu.ec

(iD) https://orcid.org/0000-0001-5047-2629

Luis Fernando Xavier Núñez-Sotomayor

Universidad Central del Ecuador

Quito, Ecuador

Ifnunezs@uce.edu.ec

https://orcid.org/0000-0002-8944-012X

Carlos Efrén Ávila-Quinga Instituto Tecnológico Honorable Consejo Provincial de Pichincha

Quito, Ecuador

cavila@tecnologicopichincha.edu.ec https://orcid.org/0000-0001-8960-2988

Pablo Anthony Rendón-Morales Universidad Central del Ecuador

Quito, Ecuador parendon@uce.edu.ec https://orcid.org/0000-0003-0448-3620

Recibido • Received • Recebido: 10 / 05 / 2019

Corregido • Revised • Revisado: 27 / 05 / 2020

Aceptado • Accepted • Aprovado: 01 / 08 / 2020 
http://doi.org/10.15359/ree.24-3.14

http://www.una.ac.cr/educare

educare@una.ac.cr

Resumen: El objetivo del artículo fue presentar un Modelo de aprendizaje microcurricular de Educación Física, con base en una revisión bibliográfica de 33 textos académicos resultantes del proceso de criterios de inclusión y exclusión, que se enmarcó en un período de publicación correspondientes a 1994 hasta el 2020, de las bases de datos Dialnet, Google Scholar y la plataforma del Ministerio de Educación del Ecuador. Se analizaron cuatro dimensiones: Currículo de Educación Física ecuatoriano, enfoques curriculares, metodologías activas y la contextualización de contenidos. Esto contribuyó a la construcción de una propuesta curricular que direccione al estudiantado hacia el desarrollo de sus destrezas motrices, sociales, cognitivas y afectivas, desde la transversalidad de los enfoques curriculares inclusivo, lúdico y de la corporeidad; y, que sirva de una guía de planificación que asegure la autonomía del estudiantado para que pueda replicar diversos ejercicios físicos durante toda su vida.

Palabras claves: Currículo; educación física; estrategia de aprendizaje; método activo; solución de problemas.

Abstract: This article aimed to describe a microcurricular learning model of Physical Education; the description was based on a bibliographic review of 33 academic texts resulting from the process of inclusion and exclusion criteria. This process was framed in a publication period corresponding to 1994 until 2020, from the Dialnet, Google Scholar databases, and the platform of the Ministry of Education of Ecuador. Four dimensions were analyzed: Ecuadorian Physical Education Curriculum, curricular approaches, active methodologies, and contextualization of content. This analysis contributed to the construction of a curricular proposal that directs students to the development of their motor, social, cognitive, and affective skills, under the mainstreaming of the inclusive, playful, and corporality curricular approaches; it also serves as a planning guide that ensures the autonomy of the students so that they can replicate various physical exercises throughout their lives.

Keywords: Active method; physical education; curriculum; learning strategy; problem solving.

Resumo: Oobjetivo do artigo foiapresentar um modelo de aprendizagem micro curricularde Educação Física, baseado em uma revisão bibliográfica de 33 textos acadêmicos resultantes do processo de critérios de inclusão e exclusão, enquadrados em um período de publicação correspondente a 1994 até 2020, das bases de dados Dialnet, Google Scholar e da plataforma do Ministério da Educação do Equador. Foram analisadas quatro dimensões: currículo de educação física do Equador, abordagens curriculares, metodologias ativas e contextualização de conteúdo. Isso contribuiu para a construção de uma proposta curricular que direciona os alunos ao desenvolvimento de suas habilidades motoras, sociais, cognitivas e afetivas, sob a integração das abordagens curriculares inclusivas, lúdicas e corporais. Serve como um guia de planejamento que garante a autonomia dos alunos para que eles possam replicar vários exercícios físicos ao longo de suas vidas.

Palavras-chave: Currículo; educação física; estratégia de aprendizagem; método ativo; resolução de problemas. 
http://doi.org/10.15359/ree.24-3.14

http://www.una.ac.cr/educare educare@una.ac.cr

\section{Introducción}

La enseñanza de la Educación Física en las instituciones educativas ecuatorianas se ha desarrollado a través de los años desde dos modelos educativos claramente identificados, como son el conductismo y el tradicionalismo.

Dentro de las teorías de motivación extrínsecas del conductismo, Skinner menciona el condicionamiento operante como una conducta dada por elementos externos (Skinner, 1987); en el caso del estudiantado de educación física la conducta se daría como respuesta a estímulos verbales y visuales emitidos por el personal docente, en el que explica y demuestra los ejercicios o actividades a realizar, en muchos de los casos enfocados a la deportividad y al desarrollo de las capacidades físicas; al respecto, el estudiantado solo tiene la posibilidad de repetir e imitar gestos técnicos y acciones motrices.

El modelo tradicionalismo militar se simplifica en que los futuros grupos docentes de educación física adquirieron sólidos conocimientos sobre actividad física y deporte durante su servicio militar obligatorio (Congreso Nacional del Ecuador, 1994) y los transmiten a sus estudiantes en todos los niveles educativos, con el resultado de sesiones-clase de educación física con disciplina militarizada, que se apoya con voces de mando para orientar clases rutinarias y mecánicas.

En el 2016 se realizó el ajuste del currículo de educación física ecuatoriano, fundamentado por el modelo educativo constructivista de aplicación obligatoria en todo el sistema educativo del país (Ministerio de Educación Ecuador, 2017). León Quinapallo et al. (2020) definen este currículo "como una propuesta innovadora, abierta y flexible, en que el futuro bachiller estará en condiciones de ser autónomo de la práctica de la actividad física, planteado desde el perfil del bachiller ecuatoriano, en [sic] base a la necesidad de la sociedad" (p. 271). Éste se basa en tres enfoques curriculares: el de la construcción de la corporeidad, el lúdico y el inclusivo. Pereira Valdez et al. (2020, p. 186) mencionn que este currículo es abierto debido a que "los docentes podrán contextualizar los contenidos curriculares".

Este cambio curricular hizo que las metodologías de enseñanza aprendizaje derivadas de los modelos educativos conductista y tradicionalista dejaran de ser funcionales y aplicables, debido a que el estudiantado debe construir su entendimiento del contenido curricular, relacionarlo con su realidad y, a la par, desarrollar capacidades de cognitivas, sociales y afectivas. Por tanto, se hizo necesario que el personal docente obtuviera otras alternativas de enseñanza, como las metodologías activas, donde el estudiantado tiene la posibilidad de aprender por sí mismo de acuerdo con sus características y potencialidades; además, el rol docente cambió de proponer la imitación y la repetición de actividades a la adopción de nuevas características como orientar, mediar, facilitar, guiar e innovar (Hernández, 2008).

Estos antecedentes expuestos han motivado el diseño de un modelo de aprendizaje microcurricular activo de educación física, que responda al propósito del aprendizaje de acuerdo con 
http://doi.org/10.15359/ree.24-3.14

http://www.una.ac.cr/educare

educare@una.ac.cr

el contexto mismo del estudiantado, dirigido a estimular y a mejorar la autonomía para la ejecución de ejercicios físicos, y aportar a la reducción de los altos índices de sedentarismo que registra el país.

\section{Metodología}

La metodología que se utilizó para la revisión bibliográfica fue el análisis documental, que implicó la consulta de 55 textos académicos clasificados en: artículos de revisión, libros, tesis de maestría, guías metodológicas, acuerdos e informes ministeriales; las bases de datos Dialnet, Google Scholar, y la plataforma del Ministerio de Educación del Ecuador. Después se realizó un proceso de criterios de inclusión y exclusión, que se enmarcó en documentos publicados en el período correspondientes a 1994 hasta el 2020 y cuyas temáticas se vinculen con cuatro dimensiones: currículo de educación física ecuatoriano, enfoques curriculares, metodologías activas y la contextualización de contenidos; como resultado se obtuvieron 33 documentos académicos directamente relacionados con las temáticas planteadas, que fueron organizados y analizados, los cuales fundamentaron el diseño de la propuesta de planificación áulica de educación física denominada Modelo de aprendizaje microcurricular activo.

\section{Estado de la cuestión}

\section{Aproximación al Currículo de Educación Física ecuatoriano}

El currículo de Educación Física del Ecuador nace a partir de la publicación del Acuerdo Ministerial 020A el 17 de febrero del 2016 (Ministerio de Educación Ecuador, 2016) como resultado de la combinación del Programa Aprendiendo en Movimiento que permitía el desarrollo integral del estudiantado para la formación activa y saludable de una ciudadanía autónoma que aportara positivamente a la sociedad (Ministerio de Educación Ecuador, 2014) y el Currículo Nacional de Educación Física 2012 que, por primera vez, planteaba el desarrollo de las destrezas cognitivas, sociales y afectivas en un ámbito de creatividad y de construcción motriz (Ministerio de Educación Ecuador, 2012).

El modelo educativo que guía este currículo es el constructivista social que, según Pinto Ladino et al. (2019) es "el proceso de formación de las funciones psicológicas superiores, se dará a través de la actividad práctica e instrumental, pero no individual, sino en la interacción o cooperación social" (p. 121); en este mismo sentido, Delgado Cedeño et al. (2018) afirman que "el currículo de los niveles de educación obligatoria se basa en los principios pedagógicos del Socio-Constructivismo, ya que favorece que los estudiantes construyan significados y sentidos, durante un proceso de aprendizaje basado en la interacción" (p. 53). En la misma línea, León Quinapallo et al. (2020) plantean que el modelo asegura "por medio de la praxis niveles altos en la calidad educativa y la autonomía investigativa y de adquisición de conocimientos, para desarrollarse como entes creativos, proactivos y emprendedores en una sociedad cada vez cambiante" (p. 272). Posso Pacheco (2018a) asevera que el modelo educativo que mejor respondía a la expresión de la sociedad y al currículo fue el constructivista social.

Richar Jacobo Posso-Pacheco, Laura Cristina Barba-Miranda, Ángel Freddy Rodríguez-Torres, Luis Fernando Xavier Núñez-Sotomayor, Carlos Efrén Ávila-Quinga y Pablo Anthony Rendón-Morales 
http://doi.org/10.15359/ree.24-3.14 http://www.una.ac.cr/educare educare@una.ac.cr

A través del análisis de los enunciados, se puede determinar que este currículo permite la construcción del conocimiento motriz, a través de una serie de técnicas y estrategias para adquirir el conocimiento previo del estudiantado, el cual generarála conexión directa con el nuevo conocimiento, que será guiado por el personal docente hasta la construcción cooperativa de la actividad o ejercicio; en la cual se entrelazan valores, posibilidades de solución, elección, acuerdos y distribución con el objetivo final del aprendizaje. No cabe duda de que con este lineamiento la educación física cuenta con pocas estrategias metodológicas que permitan desarrollar a plenitud este currículo.

\section{Aproximación a los enfoques curriculares}

Antes del 2016 en Ecuador, al igual que en algunos países latinoamericanos, los enfoques curriculares de Educación Física eran el militar, el deportivo y el de desarrollo funcional; todos ellos orientaban los contenidos curriculares hacia el cumplimiento de órdenes, de rigidez de las clases, de la competencia, del perfeccionamiento del gesto técnico y del desarrollo físico (Secretaría de Educación Pública [SEP], 1994). Sin embargo, con la emisión del nuevo currículo de Educación Física se plantearon nuevos enfoques curriculares que dieron un giro de 360 grados a la asignatura, la cual lo catapultó hacia un nuevo nivel dirigido hacia un ideal de sociedad; esos enfoques son la construcción de la corporeidad, el lúdico y el inclusivo, que generaron una visión más apegada a la contextualización de la realidad de cada institución educativa y a las necesidades y características del estudiantado, para que todo él se desarrolle en un entorno de aprendizaje (ver Figura 1).

Figura 1: Enfoques Curriculares de Educación Física del Ecuador

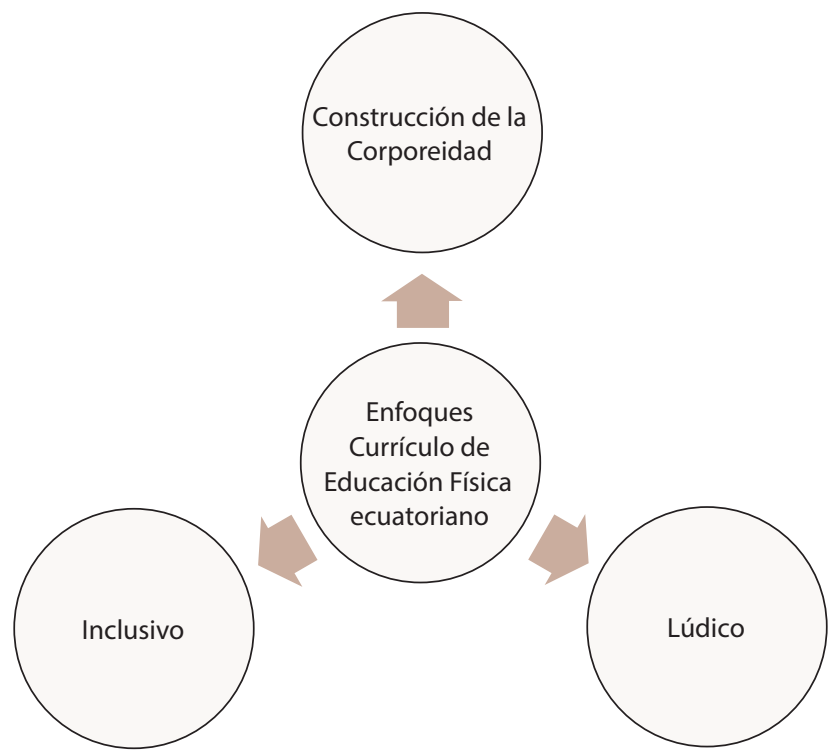

Nota: Elaboración propia con datos de la Guía de Estrategias Metodológicas de Educación Física de (Posso Pacheco, 2018a). 
http://doi.org/10.15359/ree.24-3.14

http://www.una.ac.cr/educare

educare@una.ac.cr

El enfoque de construcción de la corporeidad plantea que, en todas las clases de educación física, el estudiantado debe construirse corporalmente con el desarrollo de las capacidades físicas como las cualidades condicionantes, cualidades perceptivo-motrices y las cualidades resultantes; de acuerdo con su edad cronológica, a través de actividades estructuradas por el personal docente, con el propósito de que aflore la creatividad, la imaginación, la cognición, la sociabilidad y lo emocional, y basadas en las destrezas con criterio de desempeño pertenecientes a los seis bloques de Educación Física. Por otra parte, Hurtado Herrera (2008) menciona que la existencia en una clase de la generación de la corporeidad debe vincularse a una construcción social e intercultural.

También el enfoque lúdico direcciona a que todas las actividades realizadas en la hora de educación física deben ser divertidas y placenteras, brindar ambientes de seguridad, cuidado de si y de las demás personas; permite la exploración, el desarrollo de valores, emociones y relaciones interpersonales; promueve el aprendizaje significativo. Siempre con la orientación del personal docente que garantizará en todo momento este enfoque (Posso Pacheco, 2018a). En este mismo sentido, del Jesús Arrocha (2018) menciona que la aplicación del enfoque lúdico permite que afloren diversos valores y habilidades sociales el estudiantado, al convertir la clase en más dinámica y generadora de aprendizajes.

La inclusividad en la educación es la que permite la igualdad entre el estudiantado con diversas habilidades para poderse desarrollar en sus actividades escolares (Erkilic y Durak, 2013). Puigdellívol Aguadé (2014) también afirma que la inclusión cambia el estilo de la clase al facilitar la participación y logro curricular de todo el estudiantado; en este sentido, el enfoque inclusivo del currículo de Educación Física ecuatoriano permite que todo el estudiantado pueda participar de las sesiones clases, sin que las diferencias en las capacidades físicas, técnicas, tácticas, estilos de aprendizajes, características, necesidades educativas especiales asociadas o no a la discapacidad influyan en su participación activa, sino más bien lo enriquezca, debido a que valora ampliamente lo motriz, lo cognitivo, lo social y lo emocional (Posso Pacheco, 2018b) Lleixà et al. (2017) afirma esta posición al plantear que la inclusión "favorece el desarrollo del alumnado desde sus diferentes dimensiones física, cognitiva, emocional y afectiva, además de ser beneficiosa para la mejora de la salud a lo largo de la vida" (p. 279), es decir, este enfoque permite abrir las posibilidades de integración a una clase motriz que antiguamente era una oportunidad impensable.

Todos estos enfoques curriculares tienen que ser transversalizados y aplicados en cada actividad y ejercicio de la clase de Educación Física, a través de estrategias metodológicas activas propias del área en el contexto curricular; lo cual proyectará en el estudiantado el sentido de autonomía como lo plantea Posso Pacheco, Barba Miranda, Marcillo Ñacato et ál. (2020b): "la aplicación de enfoques curriculares en las clases de Educación Física genera directamente 
http://doi.org/10.15359/ree.24-3.14

la autonomía en el estudiante para la práctica de ejercicios físicos pudiendo ser replicados en todos los ámbitos en que se desarrollan" (p. 143). Al respecto, se necesita establecer un direccionamiento claro sobre la estructura de una clase en la que se logren fusionar y desarrollar los cuatro enfoques curriculares.

\section{Aproximación a las metodológicas activas de Educación Física}

Las metodologías activas aplicadas en el aula ayudan al profesorado a generar estrategias metodológicas innovadoras y de calidad, centradas específicamente en las necesidades y características del estudiantado, enriqueciéndoles con conocimientos formales y no formales que les servirán para que puedan desarrollar su autonomía y se desarrollen positivamente en la sociedad. López Noguero (2005) menciona que las metodologías son un proceso de interacciones positivas entre docente-estudiante, estudiante-recurso y estudiante-contexto, las cuales permiten satisfacer las expectativas educacionales. En este mismo sentido, el personal docente debe considerar cambiar su forma de enseñanza, pues el estudiantado no es el mismo del último lustro, es decir, no se debe concebir al estudiantado como pasivo ni activo, sino se debe centrar en sus necesidades de acuerdo con el medio en que se desarrolle; esto implica cambiar los objetivos, contenidos de aprendizaje y metodologías (Murillo Estepa, 2007).

La Educación Física también se ha ido orientando a la aplicación de metodologías activas, debido a que persigue nuevos retos necesarios para el desarrollo del estudiantado actual. López Pastor et al. (2016) afirman que, para dar una respuesta efectiva e innovadora, el docente o la docente tiene que trabajar en investigación en el aula, solo así se podrá adaptar o crear estrategias metodológicas apropiadas al estudiantado. Aportando a esta idea, Kirk (2008) considera que la transferencia de los conocimientos adquiridos en la escuela deben ser útiles para la vida, deben relacionarse con el medio en que se desarrollará el estudiantado y las instituciones educativas deben tener la visión de transformación social, por lo cual es prioritario contextualizar las metodologías de Educación Física.

Cada vez son más las investigaciones que se han realizado referentes a la aplicación de metodologías activas en Educación Física y cada vez los resultados coinciden claramente en que "existe otra forma de hacer educación, que sólo es necesario desearlo, visualizarlo, creer que una escuela mejor es posible y trabajar duro para conseguirlo" (Nielsen Rodríguez et al., 2020, p. 503). Estas investigaciones están sirviendo de base fundamental para las adaptaciones metodológicas a la diversidad y a la complejidad de un mundo globalizado, que requiere cubrir necesidades y expectativas educacionales inciertas. Con este lineamiento los cuerpos docentes deben estar claros en que el único camino es tomar decisiones prontas, las cuales les permitan innovar en el aula. Su experiencia, combinada con lo anterior, generará líderes docentes, no solo en el ámbito educativo, sino social, al aportar directamente a esta problemática. Esta 
http://doi.org/10.15359/ree.24-3.14

http://www.una.ac.cr/educare

educare@una.ac.cr

afirmación se complementará con la motivación docente para su formación continua, que le ayudará a buscar la innovación de la transformación educativa (Cargua García et al., 2019), para lo cual tendrá que desarrollar también habilidades investigativas que sustenten sus alternativas metodológicas como incremento de sus niveles profesionales (Rodríguez-Torres et al., 2018).

El aprendizaje cooperativo de Educación Física se considera como una metodología activa innovadora desde finales de los noventa y principio del 2000 (Velázquez Collado, 2004), a partir de ahí aparecen muchas publicaciones sobre la práctica de la educación física cooperativa como recurso metodológico; además, en España, se organiza el primer congreso de actividades físicas cooperativas en el cual se pueden compartir experiencias, investigaciones y discusiones científicas (Velázquez Colladoł, 2013). En este sentido, la lógica de la cooperación nace en respuesta a las necesidades de una nueva educación física que va en concordancia con el cambio de una sociedad del nuevo milenio; como aporte a esta transformación se puede mencionar a Velázquez Collado (2015) para quien la lógica de cooperación se debe plantear a través de técnicas específicas que permitirán su estructuración sistémica con el apoyo de un enfoque de coopedagogía.

Por otra parte, en Latinoamérica comienzan a aparecer brotes transformadores en la Educación Física, primero con la introducción en las sesiones clases de juegos cooperativos, después con actividades cooperativas y en los últimos quince años con el aprendizaje cooperativo, como una propuesta superior del área en la que se consideran las necesidades del estudiantado para desarrollar las habilidades físicas, su desenvolvimiento social, su aspecto cognitivo, su desarrollo afectivo, su vinculación con la salud, su conciencia de corporeidad, su promoción de valores, entre otros; es decir, la formación de estudiantes para que contribuyan positivamente a la sociedad como seres activos y saludables (Hernández Fernández y Madero Valencia, 2008).

Como complemento del aprendizaje cooperativo, los desafíos cooperativos se estructuran como parte fundamental del quehacer educativo; esto, es debido a que en las actividades se plantea previamente un reto que será el generador de la sesión clase, éste deberá ser superado por el estudiantado como un planteamiento docente de problema motriz a resolver, apoyado de cuatro elementos: el conceptual, el motriz, el social y el afectivo, que deberán ser trabajados integralmente (Fernández-Río y Velázquez Collado, 2005). Además, generan situaciones de aprendizaje en las que, solo dialogando, poniéndose de acuerdo, tomando decisiones, cumpliendo lo asignado y cooperando con un solo objetivo pueden resolver las dificultades planteadas (Velázquez Collado, 2016), es decir, se da la verdadera autonomía del estudiantado puesta en práctica, siempre con el apoyo y guía docente.

En el Ecuador, al igual que en otros países en donde primaban los enfoques deportivista, de capacidad física y militar, las metodologías activas de educación física eran vistas como 
http://doi.org/10.15359/ree.24-3.14

generadoras de indisciplina, desorganización, juego, actividades infructuosas y una pérdida de tiempo (Solé i Gallart, 1997), en oposición a lo que realmente son las metodologías activas y su gran importancia en el desarrollo interpersonal, cognitivo, afectivo y físico, a través de la construcción de su corporeidad.

En este aspecto, la primera información que se obtuvo sobre la implementación del currículo de Educación Física y las metodologías activas fue en el Informe 003-2017 del proceso de socialización meso y microcurrilar de Educación Física del 15 de diciembre del 2017, emitido por la Dirección Nacional de Currículo del Ministerio de Educación, en la que se concluye que se socializó a 1110 docentes del área, quienes necesitan apoyo para dominar los enfoques metodológicos para concretarlos en estrategias aplicables en las sesiones clase (Dirección Nacional de Currículo, Ministerio de Educación, 2017).

De la misma forma, se obtuvo información sobre la implementación del currículo de Educación Física y las metodologías activas con el Informe MINEDUC-DNC-0030-2018 Plan Esmeraldas "Fortalecimiento del Currículo de Educación Física del 17 de octubre del 2018, emitido por la Dirección Nacional de Currículo en la que se concluye que se socializó a 804 docentes de Educación Física, los cuales fortalecieron sus conocimientos curriculares, pero se necesita profundizar en metodologías activas de educación física y contextualizarlas, debido a que todavía no pueden descender en el aula las metodologías ni los enfoques propuestos (Dirección Nacional de Currículo, Ministerio de Educación, 2018).

A finales del 2018, el Ministerio de Educación, a través de la Dirección Nacional de Currículo, elabora una Guía de estrategias metodológicas de Educación Física para Educación General Básica y Bachillerato General Unificado, en la cual se emiten lineamientos a nivel microcurricular, específicamente en la estructuración de una sesión clase con apoyo de metodologías activas como el aprendizaje cooperativo, aprendizaje significativo y retos motrices. Pero ante las extensas posibilidades del currículo en ser desarrollado y la necesidad de aplicar los enfoques curriculares en las clases, se requiere de una variedad de estrategias metodológicas, que permitan contextualizar específicamente la naturaleza de la educación física ecuatoriana.

\section{Aproximación a la contextualización de contenidos}

El área de Educación Física tiene cinco horas pedagógicas a la semana de $1^{\circ}$ a $10^{\circ}$ de Educación General Básica y de dos horas pedagógicas a la semana de $1^{\circ}$ a $3^{\circ}$ de Bachillerato General Unificado (Ministerio de Educación Ecuador, 2016). En el currículo de Educación Física se desarrollan seis bloques curriculares, de los cuales cuatro bloques son principalmente determinados para prácticas lúdicas, gimnásticas, expresivo comunicativas y deportivas; y dos bloques transversales referidos a la identidad corporal y relación existente entre las prácticas 
http://doi.org/10.15359/ree.24-3.14

http://www.una.ac.cr/educare

educare@una.ac.cr

corporales y la salud, cada una de estas con diferentes destrezas y con criterios de desempeño o contenidos curriculares; con la particularidad de que las planificaciones microcurriculares por unidad didáctica involucran destrezas con criterio de desempeño de tres a seis bloques curriculares diferentes (Ministerio de Educación Ecuador, 2017).

Estas destrezas con criterio de desempeño deben ser desagregadas, gradadas, seleccionadas y excluidas en el proceso de planificación didáctica, en su contenido curricular y en su nivel de complejidad, para garantizar la jerarquización y contextualización según el propósito del aprendizaje del estudiantado en pos de cumplir con el perfil de salida del bachillerato ecuatoriano. Sobre ello, León Quinapallo et al. (2020) mencionan que la destreza con criterio de desempeño tiene cuatro elementos"la destreza que no es necesariamente motriz sino también cognitiva, y que es el generador de todas las actividades propuestas en ellas; el nivel de complejidad es la dificultad ... y el contexto" (p. 275). Posso Pacheco, Barba Miranda, León Quinapallo et al. (2020) también mencionan que el trabajo de investigación realizado "contribuyó a conocer la necesidad de interpretar y contextualizarlas destrezas con criterio de desempeño, comotambién la importancia de aplicar metodologías activas para garantizar que el aprendizaje sea significativo y la autonomía del estudiante en la práctica de actividad física" (p. 378). Con esto se afirma que se encuentra latente la necesidad de diseñar un esquema microcurricular que se alinee a la complejidad del currículo ecuatoriano.

\section{Discusiones}

Después de realizar el análisis anterior, se hace necesario plantear una propuesta de planificación áulica de Educación Física que se contextualice en el currículo ecuatoriano y sus enfoques, la cual sea el resultado de hibridar el aprendizaje cooperativo, el aprendizaje significativo y los desafíos motrices, tomando como base el modelo de sesión clase planteada en la Guía de estrategias metodológicas de Educación Física para EGB y BGU (Posso Pacheco, 2018a) y la Propuesta de estrategias metodológicas aplicadas a la Educación Física (Posso Pacheco, 2018b), con la finalidad de alcanzar el perfil de salida exigida del bachillerato ecuatoriano, en el que todo el estudiantado desarrolle valores, principios y liderazgo, a través de poder construir, buscar alternativas de solución, asignar responsabilidades, trabajar en equipo, resolver problemas, ejecutar, y superar desafíos motrices, cognitivos, sociales y afectivos.

Se plantea el modelo de aprendizaje microcurricular activo que constará de tres fases: apertura, desarrollo y cierre de clase (Posso Pacheco, 2018a); sin embargo, los momentos, técnicas y estrategias se diferenciarán para garantizar la aplicabilidad áulica del modelo, esenciales para que el estudiantado pueda adquirir la capacidad de replicar lo aprendido en su vida diaria. La propuesta se detalla a continuación (ver Tabla 1). 
http://doi.org/10.15359/ree.24-3.14

Tabla 1: Modelo de aprendizaje microcurricular activo para Educación Física

\begin{tabular}{|c|c|c|}
\hline Fases & Momentos & Técnicas y estrategias \\
\hline \multirow[t]{5}{*}{ 1. Apertura } & 1. Organización del estudiantado & Técnicas de agrupamiento, asociación, divisorias y aleatoria. \\
\hline & \multirow[t]{2}{*}{ 2. Conocimiento previo } & $\begin{array}{l}\text { Estrategias preinstruccionales: consultas, investigaciones, } \\
\text { lecturas previas, videos, observaciones. }\end{array}$ \\
\hline & & $\begin{array}{l}\text { Técnicas: Lluvia de ideas, actividad focal introductoria, } \\
\text { discusión guiada. }\end{array}$ \\
\hline & 3. Objetivo & $\begin{array}{l}\text { Técnica de exposición y estrategias de Involucramiento } \\
\text { grupal. }\end{array}$ \\
\hline & 4. Juegos de activación & Técnicas calisténica y lúdica de grupo. \\
\hline \multirow[t]{2}{*}{ 2. Desarrollo } & 5. Desafío & Planteamiento del desafío motriz \\
\hline & 6. Desarrollo & $\begin{array}{l}\text { Comprensión del reto } \\
\text { Formulación de preguntas de desarrollo } \\
\text { Selección de soluciones } \\
\text { Asignación de responsabilidades } \\
\text { Ensayo y error sistemático }\end{array}$ \\
\hline 3. Cierre & 7. Autonomía del proceso & $\begin{array}{l}\text { Concreción de momentos } \\
\text { Cumplimiento de fines } \\
\text { Construcción del conocimiento } \\
\text { Proceso de soluciones de la actividad }\end{array}$ \\
\hline
\end{tabular}

Nota: Elaboración propia con datos de la Guía de estrategias metodológicas de Educación Física (Posso Pacheco, 2018a).

\section{Fase 1. Apertura}

Momento 1. Organización del estudiantado: El primer paso antes de iniciar la clase de Educación Física es la organización del estudiantado, la cual permite múltiples beneficios para docente y estudiantes. Este momento es crucial para el direccionamiento de la clase, por lo que no debe ser rígida ni monótona; se deben aplicar técnicas que permitan la transversalidad de los enfoques curriculares de Educación Física, mediante la utilización de técnicas de agrupación, asociación, divisorias, y de selección aleatoria que dividan la clase en grupos heterogéneos de tres a siete estudiantes, según el objetivo planteado, nivel o edad.

Momento 2. Conocimiento previo: una vez que se ha organizado al estudiantado por grupos de trabajo, se deben activar sus conocimientos previos o experiencias vividas referentes al tema a desarrollar; para esto cada docente utilizará varias estrategias preinstruccionales a manera de trabajo investigativo o de consulta previa extraescolar; genera un primer acercamiento a lo que se va a desarrollar, por ejemplo, con lecturas, videos, observaciones, preguntas, entre 
http://doi.org/10.15359/ree.24-3.14

http://www.una.ac.cr/educare

educare@una.ac.cr

otras. También aplicará técnicas como lluvia de ideas, actividad focal introductoria y discusión guiada, las cuales permitirán que el estudiantado active sus recuerdos y se motive por las expectativas por conocer.

Momento 3. Objetivo: Es importante que el docente o la docente exponga al estudiantado los objetivos de la clase después de haber generado en ellos diferentes conocimientos previos, lo cual permitirá conocer la utilidad y los beneficios que conlleva la clase, y crear espacios de compromiso para alcanzar los propósitos planteados través de estrategias de involucramiento en las diferentes actividades por realizar.

Momento 4. Juegos de activación: Con el involucramiento del estudiantado para el cumplimiento del objetivo de clase, se debe desarrollar una serie de ejercicios, juegos o actividades de bajo impacto, en la cual se implique a todo el organismo, con la finalidad de aumentar la disposición corporal para ejercicios más intensos por desarrollar en la fase 2. Para esto el personal docente utilizará varias técnicas como la calisténica que se aplica con el músculo frío con rotaciones, circunducciones y elongaciones cortas, dinámicas y con varias repeticiones de máximo cuatro segundos; y, las técnicas lúdicas de grupo como las sensoriales y las motoras, en las cuales se realizan ejercicios físicos de baja intensidad a través del juego; éstas deben ser adaptadas a la edad, característica y nivel del estudiantado, como también deben ser motivadoras y una introducción a lo que se va a desarrollar en la siguiente fase.

\section{Fase 2. Desarrollo}

Momento 5. Desafío: Una vez que se ha realizado la introducción, en el momento juegos de activación, se procederá a plantear el desafío motriz, el cual debe ser diseñado para el cumplimiento del objetivo de la clase; también debe ser propuesto de acuerdo con las destrezas con criterio de desempeño de la planificación microcurricular de unidad didáctica, y, al igual que los juegos de activación, deben ser adaptados a los niveles y subniveles de educación, edad, características y necesidades del estudiantado.

El desafío motriz es un generador de aprendizaje de la educación física, en la que el estudiantado desarrolla cooperativamente destrezas motrices, cognitivas, afectivas y sociales para alcanzar, en clase, la resolución de problemas y el cumplimiento de los enfoques del currículo nacional de Educación Física, como son la construcción de la corporeidad, el lúdico y la inclusión.

Momento 6. Desarrollo: Planteado el desafío motriz al estudiantado, el personal docente deberá direccionar el cumplimiento de cada una de las estrategias de esta fase, asumiendo un rol de mediador y orientador durante todo el proceso de enseñanza aprendizaje, a través de interacciones positivas y motivaciones permanentes de logros, como también al permitir la elección de diferentes posibilidades de ejecución y corrección de técnicas, tácticas, ejercicios y actividades. 
http://doi.org/10.15359/ree.24-3.14

a. Comprensión del reto: Cada docente debe asegurarse de que los grupos hayan entendido, comprendido y definido el problema planteado en el desafío motriz; en este sentido, el estudiantado debe saber qué persigue el reto, tener claridad de lo que tienen que hacer, tomar conciencia para su aceptación y conocer el problema que genera el desafío. Es decir, el estudiantado debe tener la capacidad de responder a las interrogantes ¿por qué?, y ¿para qué? del desafío motriz, a través de repetir verbalmente el desafío o con preguntas directas realizadas por el docente o la docente; para esto se debe tomar muy en cuenta la edad del estudiantado y el grado de complejidad del desafío.

b. Formulación de preguntas de desarrollo: Una vez que el estudiantado de cada grupo tiene claro el desafío motriz y cuál es el problema a superar; les surgirán varias inquietudes por lo que el personal docente les deberá guiar, para que formulen varias preguntas alrededor de: ¿cómo superar el reto?, esto dependerá de los niveles educativos, los contenidos y las edades del estudiantado.

c. Selección de soluciones: Los grupos de estudiantes al formular un número indeterminado de preguntas deberán responder cada una de ellas, de tal forma que tendrán varias soluciones que posteriormente se transformarán en actividades que les permitirán superar el desafío motriz, por lo que seleccionarán, democráticamente, la mejor solución para ser desarrollada.

d. Asignación de responsabilidades: Elegida la mejor solución para superar el desafío motriz, los grupos de estudiantes planificarán paso a paso las actividades por cumplir de forma cooperativa; por lo que, el docente o la docente solo orientará al estudiantado a que se organice y se distribuya autónomamente las responsabilidades por cumplir para cada tarea del desafío motriz.

e. Ensayo y error sistemático: Una vez que los grupos se hayan organizado y distribuido las tareas para el cumplimiento del desafío motriz, el docente o la docente orientará al estudiantado para que, de forma independiente y en interacción cooperativa, ejecuten lo planificado. Esto también garantizará la transversalización de los enfoques curriculares de construcción de la corporeidad, lúdica e inclusiva, a través de la estrategia ensayo error sistematizado; es decir, la actividad o tarea propuesta deberá ser construida, divertida, placentera, con participación activa motriz, cognitiva, social y afectiva de todos los sujetos integrantes del grupo, para lo cual deberán repetir varias veces las actividades propuestas $y$, al mismo tiempo, corregir los errores encontrados, lo cual realizarán de forma gradual y progresiva hasta conseguir la superación del desafío motriz. 
http://doi.org/10.15359/ree.24-3.14

http://www.una.ac.cr/educare

educare@una.ac.cr

\section{Fase 3. Cierre}

Momento 7. Autonomía del proceso: Después de que se desarrolle la fase 2, el docente debe realizar un proceso de evaluación individual y grupal del estudiantado, en el que se debe valorar el desempeño motriz, cognitivo, social y emocional de las actividades realizadas para superar el desafío motriz; estas deben concordar con los indicadores de evaluación propuestos en el currículo de Educación Física por subnivel y nivel educativo. Esta evaluación permitirá medir el desarrollo y el alcance de la autonomía del estudiantado adquirida en la sesión clase, a través de varias estrategias como son la concreción de momentos, la formulación de fines, la construcción de conocimientos, la relación de experiencias y la elección de soluciones (ver Tabla 2).

Tabla 2: Matriz de evaluación de la autonomía de procesos de Educación Física

\begin{tabular}{|l|l|l|l|l|}
\hline \multicolumn{1}{|c|}{ Estrategias } & $\begin{array}{c}\text { Concreción de } \\
\text { momentos }\end{array}$ & $\begin{array}{c}\text { Cumplimiento de } \\
\text { fines }\end{array}$ & $\begin{array}{c}\text { Construcción de } \\
\text { conocimientos }\end{array}$ & $\begin{array}{c}\text { Proceso de } \\
\text { soluciones }\end{array}$ \\
\hline Motriz & & & & \\
\hline Cognitivo & & & & \\
\hline Social & & & & \\
\hline Afectivo & & & & \\
\hline
\end{tabular}

Nota: Elaboración propia con datos de la Guía de estrategias metodológicas de Educación Física (Posso Pacheco, 2018a).

a. Concreción de momentos: El personal docente evaluará de forma individual y grupal el desarrollo de los seis momentos de las dos primeras fases del Modelo de aprendizaje microcurricular activo de Educación Física, tomando en cuenta su accionar con el desempeño de las técnicas y estrategias propuestas.

b. Cumplimiento de fines: También se evaluará la observación del desarrollo de las destrezas motrices según la edad del estudiantado y la exigencia de la actividad; desarrollo cognitivo de acuerdo con el cumplimiento táctico y estratégico que influyó en el resultado final de la actividad; desarrollo social, donde se tome en cuenta el nivel interpersonal deseado en la actividad, y desarrollo afectivo que debe considerar el nivel emocional positivo en la realización de la actividad.

c. Construcción de conocimientos: Consiste en evaluar al estudiantado según el grado de integración de los conocimientos previos, con la interacción de la actividad en relación con el contexto. 
http://doi.org/10.15359/ree.24-3.14

d. Proceso de soluciones de la actividad: Se evaluarán individual y grupalmente todos los aportes que se han realizado en torno al proceso de solución de la actividad como: comprensión, formulación, respuesta y selección de la mejor solución.

\section{Conclusiones}

Esta nueva propuesta de planificación áulica de Educación Física, denominada Modelo de aprendizaje microcurricular activo, responde al análisis bibliográfico realizado en esta investigación; será de gran importancia para el personal docente de Educación Física del Ecuador, debido a que guiará la aplicación de los contenidos curriculares contextualizados al propósito del aprendizaje, en donde los enfoques se transversalizarán y se aplicarán permanentemente, para obtener los resultados del aprendizaje esperados de acuerdo con el perfil de salida de cada subnivel educativo, al proporcionar lineamientos claros en concordancia con el currículo de Educación Física ecuatoriano y a su modelo educativo constructivista; convierte al personal docente como verdadero mediador del proceso de enseñanza aprendizaje, al lograr que el estudiantado descubra, adapte, modifique y construya su entendimiento de la corporeidad y motricidad; con lo cual generará la autonomía para la realización del ejercicio físico, como seres críticos, reflexivos, activos y saludables.

\section{Declaración de Material complementario}

Este artículo tiene disponible como material complementario:

- La versión preprint del artículo en https://doi.org/10.5281/zenodo.3534627

\section{Referencias}

Cargua García, A. X., Posso-Pacheco, R. J., Cargua García, N. I. y Rodríguez Torres, Á. F. (2019). La formación del profesorado en el proceso de innovación y cambio educativo. OLIMPIA. Revista de la Facultad de Cultura Física de la Universidad de Granma, 16(54), 140-152. http:// www.ub.edu/obipd/la-formacion-del-profesorado-en-el-proceso-de-innovacion-ycambio-educativo/

Congreso Nacional del Ecuador. (15 de septiembre, 1994). Ley de servicio militar obligatorio en fuerzas armadas nacionales. Ley 68. https://www.defensa.gob.ec/wp-content/uploads/ downloads/2015/04/feb15 LEY-DE-SERVICIO-MILITAR-OBLIGATORIO-EN-FUERZASARMADAS-NACIONALES.pdf 
http://doi.org/10.15359/ree.24-3.14

http://www.una.ac.cr/educare

educare@una.ac.cr

Delgado Cedeño, J. J., Vera Vera, M. G., Cruz Mendoza, J. C. y Pico Mieles, J. G. (2018). El currículo de la educación básica ecuatoriana: Una mirada desde la actualidad. Revista Cognosis, 3(4), 47-66. https://186.46.160.238/index.php/Cognosis/article/view/1462/1718

Del Jesús Arrocha, L. (2018). Programa para el desarrollo de habilidades socio-emocionales en alumnos de educación infantil con altas capacidades intelectuales [Tesis de maestría]. Universidad Internacional de la Rioja, Logroño. https://reunir.unir.net/ bitstream/handle/123456789/7003/DEL\%20JESUS\%20ARROCHA\%2c\%20LAURA. pdf? sequence $=1$ \&isAllowed $=y$

Dirección Nacional de Currículo, Ministerio de Educación. (2017). Informe del proceso de socialización mesocurricular y microcurricular de educación física. Autor.

Dirección Nacional de Currículo, Ministerio de Educación. (2018). PlanEsmeraldas "Fortalecimiento del currículo de Educación Física. Autor.

Erkilic, M. y Durak, S. (2013). Tolerable and inclusive learning spaces: An evaluation of policies and specifications for physical environments that promote inclusion in Turkish Primary Schools. International Journal of Inclusive Education, 17(5), 462-479. https://doi.org/10.108 $\underline{0 / 13603116.2012 .685333}$

Fernández-Río, J.y Velázquez Collado, C. (2005). Desafíos físicos cooperativos: Retos sin competición para las clases de educación física. Wanceullen.

Hernández, Y. (2008). Nuevo rol del docente del siglo XXI. https://www.gestiopolis.com/nuevo-roldel-docente-del-siglo-xxi/

Hernández Fernández, L. y Madero Valencia, O. M. (2008). El aprendizaje cooperativo como metodología de trabajo en educación física. Talleres gráficos SNTE. https://www. orientacionandujar.es/wp-content/uploads/2019/01/cooperativo-en-Educaci\%C3\%B3nF\%C3\%ADsica.pdf

Hurtado Herrera, D. R. (2008). Corporeidad y motricidad. Una forma de mirar los saberes del cuerpo. Educação \& Sociedade, 29(102), 119-136. https://www.scielo.br/pdf/es/v29n102/ a0729102.pdf

Kirk, D. (2008, Octubre). Los futuros de la educación física: La importancia de la cultura física y de la "idea de la idea" de educación física. En Libro de Actas V Congreso Internacional de la Asociación Española de Ciencias del Deporte. https://docplayer.es/34858954-Los-futurosde-la-educacion-fisica-la-importancia-de-la-cultura-fisica-y-de-la-idea-de-la-idea-deeducacion-fisica.html

León Quinapallo, X. P., Ortiz Bravo, N. A. y Manangón Pesantez, R. M. (2020). Currículo de los niveles de educación obligatoria: Una mirada reflexiva desde el hacer docente. Revista educare, 24(1), 270-280. https://doi.org/10.46498/reduipb.v24i1.1246 
http://doi.org/10.15359/ree.24-3.14

http://www.una.ac.cr/educare educare@una.ac.cr

Lleixà, T., Ríos, M., Gómez-Zepeda, G., Petreñas, C. y Puigdellívol, I. (2017). Actitudes inclusivas en la formación del profesorado de educación física. Aportaciones desde los Talleres de Acción Directa. Ágora para la educación física y el deporte, 19(2-3), 277-297. https://doi. org/10.24197/aefd.2-3.2017.277-297

López Noguero, F. (2005). Metodologías participativas en la enseñanza universitaria. Narcera.

López Pastor, V. M., Pérez Brunicardi, D., Manrique Arribas, J. C. y Monjas Aguado, R. (2016). Los retos de la educación física en el siglo XXI. Retos, 29, 182-187. https://recyt.fecyt.es/index. $\mathrm{php/retos/article/view/42552/25499}$

Ministerio de Educación Ecuador. (2012).Currículo de educación física para la educación general básica y bachillerato. Autor.

Ministerio de Educación Ecuador. (2014). Aprendiendo en movimiento. Programa escolar de actividad física. Medios Públicos EP.

Ministerio de Educación Ecuador. (17 de febrero de 2016). Acuerdo Ministerial 020A. Autor.

Ministerio de Educación Ecuador. (2017). Currículo de los niveles de educación obligatoria. Medios Públicos EP.

Murillo Estepa, P. (2007). Nuevas formas de trabajar en clase: Metodologías activas y colaborativas en el desarrollo de competencias docentes en la formación del profesorado. Instituto Superior de Formación del Profesorado. https://idus.us.es/xmlui/handle/11441/61247

Nielsen Rodríguez, A., Romance García, Á. R., y Chinchilla Minguet, J. L. (2020). Los ambientes de aprendizaje como metodología activa promotora de la actividad física en educación infantil. Un estudio de caso. Retos, 37, 498-504. https://recyt.fecyt.es/index.php/retos/ article/view/71026

Pereira Valdez, M. J., Velastegui, C. F. y Paz Viteri, B. S. (2020). Prácticas deportivas y enfoques curriculares: Una perspectiva desde la educación física ecuatoriana. Revista EDUCARE, 24(1), 180-197. https://doi.org/10.46498/reduipb.v24i1.1233

Pinto Ladino, J. E., Castro Bello, V. A.y Siachoque Castillo, O. M. (2019). Constructivismo social en la pedagogía. Educación y Ciencia, 22, 117-133. https://revistas.uptc.edu.co/index.php/ educacion y ciencia/article/view/10042

Posso Pacheco, R. J. (2018a). Guía de estrategias metodológicas para educación física en EGB y BGU. Ministerio de Educación Ecuador. https://www.academia.edu/38540331/Gu\%C3\%ADa de estrategias Metodol\%C3\%B3gicas de Educaci\%C3\%B3n F\%C3\%ADsica.pdf

Posso Pacheco, R. J. (2018b). Propuesta de estrategias metodológicas activas aplicadas a la educación física [Tesis de maestría]. Universidad Internacional de la Rioja, Logroño. https:// reunir.unir.net/handle/123456789/7439 
http://doi.org/10.15359/ree.24-3.14

http://www.una.ac.cr/educare

educare@una.ac.cr

Posso Pacheco, R. J., Barba Miranda, L. C., León Quinapallo, X. P., Ortiz Bravo, N. A., Manangón Pesantez, R. M. y Marcillo Ñacato, J. C. (2020a). Educación física significativa: Propuesta para la contextualización de contenidos curriculares. PODIUM. Revista de Ciencia y Tecnología en la Cultura Física, 15(2), 371-381. http://podium.upr.edu.cu/index.php/podium/article/view/914

Posso Pacheco, R. J., Barba Miranda, L. C., Marcillo Ñacato, J. C., Acuña Zapata, M. C. y Hernández Hernández, F. R. (2020b). Enfoques curriculares como contribución para la autonomía de la actividad física. EmásF:Rrevista Digital de Educación Física, 11(63), 132-145. https://dialnet. unirioja.es/servlet/articulo?codigo $=7279815$

Puigdellívol Aguadé, I. (2014). La inclusión: Aproximación conceptual. En M. Ríos Hernández, P. Ruiz Sánchez y N. C. Gres (Coords.), La inclusión en la actividad física y deportiva: La práctica de la educación física y deportiva en entornos inclusivos (pp. 5-17). Paidotribo.

Rodríguez-Torres, Á. F., Posso-Pacheco, R. J., De la Cueva-Constante, R. R. y Barba-Miranda, L. C. (2018). Herramientas metodológicas para el desarrollo de habilidades investigativas en los estudiantes. Una praxis necesaria. Olimpia: Publicación científica de la Facultad de Cultura Física de la Universidad de Granma, 15(50), 119-132. https://revistas.udg.co.cu/index.php/ olimpia/article/view/93/118

Secretaría de Educación Pública.(1994). Programa de educación física. Autor. https://es.slideshare. net/fairygaviotita/programa-educfsica1994

Skinner, B. F. (1987). Sobre el conductismo. Planeta de Agostini.

Solé i Gallart, I. (1997). Reforma y trabajo en grupo. Cuadernos de Pedagogía, 255, 50-53.

Velázquez Collado, C. (2004). Las actividades físicas cooperativas. Una propuesta para la formación en valores a través de la educación física en las escuelas de educación básica. Secretaría de Educación Pública.

Velázquez Collado, C. (2013). Análisis de la implementación del aprendizaje cooperativo durante la escolarización obligatoria en el área de educación física [Tesis doctoral]. Universidad de Valladolid, Espñaña. https://uvadoc.uva.es/bitstream/10324/2823/1/TESIS312-130521.pdf.pdf

Velázquez Collado, C. (2015). Aprendizaje cooperativo en educación física: Estado de la cuestión y propuesta de intervención. Retos, 28, 234-239. https://recyt.fecyt.es/index.php/retos/ issue/view/2040

Velázquez Collado, C. (2016). Desafíos físicos cooperativos: Relato de una experiencia. Tándem. Didáctica de la Educación Física, 53, 54-59. 